\title{
Author Correction: Predicting the clinical impact of human mutation with deep neural networks
}

Laksshman Sundaram (D), Hong Gao, Samskruthi Reddy Padigepati (D, Jeremy F. McRae (D), Yanjun LiD, Jack A. Kosmicki, Nondas Fritzilas, Jörg Hakenberg (D), Anindita Dutta, John Shon, Jinbo Xu, Serafim Batzoglou, Xiaolin Li (iD and

Kyle Kai-How Farh iD

Correction to: Nature Genetics https://doi.org/10.1038/s41588-018-0167-z, published online 23 July 2018.

In the version of this article originally published, the name of author Serafim Batzoglou was misspelled. The error has been corrected in the HTML and PDF versions of the article.

Published online: 17 December 2018

https://doi.org/10.1038/s41588-018-0329-Z

\section{Author Correction: Assembly of a pan-genome from deep sequencing of 910 humans of African descent}

Rachel M. Sherman D, Juliet Forman, Valentin Antonescu, Daniela Puiu, Michelle Daya, Nicholas Rafaels, Meher Preethi Boorgula, Sameer Chavan, Candelaria Vergara Di, Victor E. Ortega, Albert M. Levin, Celeste Eng, Maria Yazdanbakhsh D, James G. Wilson, Javier Marrugo, Leslie A. Lange, L. Keoki Williams, Harold Watson, Lorraine B. Ware, Christopher O. Olopade, Olufunmilayo Olopade, Ricardo R. Oliveira, Carole Ober, Dan L. Nicolae, Deborah A. Meyers, Alvaro Mayorga, Jennifer Knight-Madden, Tina Hartert, Nadia N. Hansel, Marilyn G. Foreman, Jean G. Ford, Mezbah U. Faruque, Georgia M. Dunston, Luis Caraballo, Esteban G. Burchard, Eugene R. Bleecker, Maria I. Araujo, Edwin F. Herrera-Paz DD, Monica Campbell, Cassandra Foster, Margaret A. Taub, Terri H. Beaty (D), Ingo Ruczinski, Rasika A. Mathias, Kathleen C. Barnes and Steven L. Salzberg (DD

Correction to: Nature Genetics https://doi.org/10.1038/s41588-018-0273-y, published online 19 November 2018.

In the version of this article initially published, the statement "there are no pan-genomes for any other animal or plant species" was incorrect. The statement has been corrected to "there are no reported pan-genomes for any other animal species, to our knowledge." We thank David Edwards for bringing this error to our attention. The error has been corrected in the HTML and PDF versions of the article.

Published online: 15 January 2019

https://doi.org/10.1038/s41588-018-0335-1

\section{Publisher Correction: Immune genes are primed for robust transcription by proximal long noncoding RNAs located in nuclear compartments}

Stephanie Fanucchi, Ezio T. Fok, Emiliano Dalla, Youtaro Shibayama, Kathleen Börner, Erin Y. Chang, Stoyan Stoychev, Maxim Imakaev DD, Dirk Grimm, Kevin C. Wang (D), Guoliang Li (D), Wing-Kin Sung and Musa M. Mhlanga (D)

Correction to: Nature Genetics https://doi.org/10.1038/s41588-018-0298-2, published online 10 December 2018

In the version of this article initially published, ' + ' and '-' labels were missing from the graph keys at the bottom of Fig. $8 \mathrm{~d}$. The error has been corrected in the HTML and PDF versions of the article.

Published online: 15 January 2019

https://doi.org/10.1038/s41588-018-0341-3 